\title{
Bestimmung der Aktivität der alkalischen Phosphatase in der Galle
}

\author{
Von M. Peterlik
}

Aus dem Institut für allgemeine und experimentelle Pathologie der Universität Wien (Vorstand: Prof. Dr. A. Lindner)

(Eingegangen am 17. Mai 1965)

\begin{abstract}
Es werden verschiedene Möglichkeiten diskutiert, um die Hemmung der alkalischen Phosphatase durch Galleninhaltsstoffe bei der Bestimmung der Aktivität auszuschalten. Dies ist nach Fraktionierung der Galle mittels Gelfiltration oder durch Ausfällung der Proteine möglich. Die genaue experimentelle Durchführung dieser Methoden wird beschrieben.
\end{abstract}

Various possibilities are discussed for abolishing the inhibitory action of bile substances on alkaline phosphatase during its estimation. This is possible after fractionation of the bile by gel filtration or by precipitation of the proteins. The exact experimental details for the method are given.

Für Untersuchungen über die Änderung von Enzymaktivitäten während der Cholerese war es wünschenswert, über eine zuverlässige Bestimmung der Aktivität der alkalischen Phosphatase („AP") in der Galle zu verfügen, bei der es zu keiner Verfälschung der Meßergebnisse durch den Einfluß von Inhibitoren kommen konnte. In verschiedenen Arbeiten war darauf hingewiesen worden, daß die AP sowohl durch Bilirubin (1) als auch durch verschiedene Gallensäuren, wie Desoxychol-, Cholsäure (2) und Taurocholsäure (3) gehemmt wird.

Eine weitere Schwierigkeit ergibt sich dadurch, daß die Gallenfarbstoffe die kolorimetrische Aktivitätsbestimmung der AP stören. Bei der von BESSEY, LOWRY und Brock (4) angegebenen Methode wird Natrium(p-nitrophenyl)-phosphat als Substrat verwendet und die Extinktion des gebildeten p-Nitrophenols bei $400 \mathrm{~m} \mu$ gemessen. Bei dieser Wellenlänge ist die Eigenabsorption der Gallenfarbstoffe immer noch beträchtlich, obwohl z. B. das Absorptionsmaximum für Bilirubin zwischen 420 und $450 \mathrm{~m} \mu$ liegt (5). Die Extinktion der als Leerwert verwendeten Galle kann selbst bei einer Verdünnung von $1: 25$ größer als die des enzymatisch gebildeten p-Nitrophenols sein, wodurch die Genauigkeit der Messung beeinträchtigt wird.

Es galt also, eine einfache und schnelle Methode zu finden, mit der es möglich ist, sowohl die als Inhibitoren für das Enzym wirkenden als auch die kolorimetrische Messung störenden Galleninhaltsstoffe zu entfernen. Dafür kamen Dialyse der Galle, Verdünnung bei gleichzeitiger Erhöhung der Substratkonzentration, Fraktionierung durch Gelfiltration oder durch Fällung der Proteine in Frage.

\section{Methodik}

Alle Untersuchungen wurden mit frischer Rindergalle ausgeführt.

\section{Fällung der Proteine mit Methanol-Ätber .}

$0,50 \mathrm{~m} l$ Galle wurden in $2,5 \mathrm{~m} l$ Methanol-Ather (peroxydfrei), $2: 3(\mathrm{v} / \mathrm{v})$, bei $-20^{\circ}$ pipettiert. Nach kräftigem Durchmischen wurde 20 Min. bei dieser Temperatur mit 3000 U/Min. zentrifugiert und der Uberstand so weit wie möglich dekantiert; die Fällung wurde in $0,3 \mathrm{~m} l$ eines $0,05 \mathrm{~m}$ Glycin-NaOH-Puffers vom $\mathrm{pH}=10,5$ (6) gelöst. Diese Lösung wurde nochmals mit $1,5 \mathrm{~m} l$ Methanol-Äther bei $-20^{\circ}$ behandelt und das Präzipitat, wie oben beschrieben, abzentrifugiert. Dann wurde es mit möglichst wenig Pufferlösung in ein $5 \mathrm{~m} l$-Kölbchen gespült. Die noch vorhandenen Spuren Methanol und Äther wurden im Vakuum bei Raumtemperatur entfernt. Die Lösung wurde dann weiter im Vakuum bei $37^{\circ}$ eingeengt, in eine graduierte Küvette gespült und mit Pufferlösung auf $1,0 \mathrm{~m} l$ aufgefüllt. $\mathrm{Je} 0,40 \mathrm{~m} l$ werden für die Bestimmung und den Leerwert entnommen.

\section{Dialyse der Galle}

$10,0 \mathrm{~m} l$ Galle wurden $24 \mathrm{Stdn}$. bei $4^{\circ}$ gegen destilliertes Wasser dialysiert, das dauernd gerührt und öfters gewechselt wurde. Dann wurde die Galle vollständig aus dem Dialysierschlauch gespült und auf $15,0 \mathrm{~m} /$ aufgefüllt.

\section{Gelfiltration}

$2,0 \mathrm{ml}$ Galle wurden an einer mit "Sephadex G-25 medium" gefüllten Säule $(1 \times 25 \mathrm{~cm})$ durch Elution mit $0,05 \mathrm{~m}$ Glycin$\mathrm{NaOH}$-Puffer $(\mathrm{pH}=10,5)$ fraktioniert. Das Eluat wurde in Fraktionen von $1 \mathrm{~m} /$ aufgefangen und die Absorption bei $280 \mathrm{~m} \mu$ bestimmt.

\section{Verdünnung}

$0,20 \mathrm{~m} l$ Galle wurden mit $4,8 \mathrm{~m} l$. Substratlösung (s. u.) versetzt, was einer Verdünnung von $1: 25$ entspricht. Die Lösung wurde 120 Min. inkubiert. Anschließend wurden $1,0 \mathrm{ml}$ entnommen, mit $0,02 n \mathrm{NaOH}$ auf $5,0 \mathrm{~m} l$ aufgefüllt und die Extinktion bei $400 \mathrm{~m} \mu$ in diesem Volumen bestimmt.

$$
\text { - }
$$

\section{Aktivitätsbestimumungen}

Alle Aktivitätsbestimmungen wurden nach der Methode von BESSEY, LOWRY und BROCK (4) durchgeführt. Als Substrat wurde Natrium-(p-nitrophenyl)-phosphat $(0,001 \mathrm{Mol}$ in $0,05 \mathrm{~m}$ Glycin$\mathrm{NaOH}-\mathrm{Puffer} ; \mathrm{pH}=10,5$ ) verwendet (Reagenzien einer „Biochemica Test=Combination" der Fa. C. F. Boehringer \& Söhne, Mannheim). Die Inkubationstemperatur betrug 37,0 $0^{\circ}$ Die Extinktion des gebildeten p-Nitrophenols wurde bei einer Wellenlänge von $400 \mathrm{~m} \mu$ in einem Spektralphotometer der $\mathrm{Fa}$. Bausch \& Lomb, Rochester, gemessen. Die Aktivität der AP wurde in Einheiten nach BESSEY, LOWRY und BROCK (4) angegeben. - Wenn nicht anders angegeben, wurden $0,20 \mathrm{~m} l$ der $\mathrm{zu}$ untersuchenden Lösung mit $1,0 \mathrm{ml}$ Substratlösung $60 \mathrm{Min}$. inkubiert; dann wurde mit $0,02 n \mathrm{NaOH}$ auf $5,0 \mathrm{ml}$ aufgefüllt und die Extinktion in diesem Volumen gemessen.

- Zur Untersuchung der Zweckmäßigkeit der angeführten Trennverfahren wurde einer Standardenzymlösung, die nach einem schon früher beschriebenen Verfahren (7) hergestellt worden war und eine Aktivität von 15 Einheiten aufwies, ein aliquoter Teil der jeweils abgetrennten Fraktionen zugesetzt, um so das Ausmaß der eventuell vorhandenen Hemmwirkung $\mathrm{zu}$ bestimmen: $0,05 \mathrm{~m} /$ der Standardenzymlösung und $0,20 \mathrm{~m} l$ der $z u$ untersuchenden Fraktion wurden mit $1,0 \mathrm{~m} /$ Substratlösung $15 \mathrm{Min}$. inkubiert. Der Leerwert bestand aus $0,20 \mathrm{~m} l$ der fraglichen Lösung und 1,0 $\mathrm{m} /$ Substratlösung. $0,05 \mathrm{~m} /$ Standardlösung wurden erst nach der Inkubation zugesetzt. 


\section{Ergebnisse}

Eine Aktivitätsbestimmung in der unbehandelten Galle ergab einen Wert von 0,10 Einheiten. Nach allen eingangs erwähnten Befunden $(2,3)$ war nicht anzunehmen, $\mathrm{daB}$ dies die "wahre" Aktivität der AP sei. Um diese Annahme zu bestätigen, wurde Galle der Standardenzymlösung zugesetzt; es trat ein Aktivitätsverlust von 13 Einheiten auf. Das Experiment zeigt deutlich, $\mathrm{da}$ die AP durch Galleninhaltsstoffe gehemmt wird. Nach der Dialy'se der Galle wurde eine Aktivität von 0,21 Einheiten gemessen. Dieser Wert liegt schon beträchtlich höher als der für die unbehandelte Galle, doch konnten die Inhibitoren unter diesen Bedingungen noch nicht vollständig entfernt werden: $0,20 \mathrm{~m} l$ der dialysierten Galle waren noch immer imstande, auf die Aktivität der Standardenzymlösung eine beträchtliche Hemmung auszuüben.

Aufgrund dieser Befunde wurde versucht, das Enzym durch Gelfiltration von den Inhibitoren zu trennen. Da die Ausschlußgrenze von "Sephadex G-25" bei einem Molekulargewicht von 5000 liegt, können Proteine auf einfache und schnelle Art von den niedermolekularen Inhibitoren und Farbstoffen getrennt werden. Eine Aktivitätsbestimmung der vereinigten Fraktionen ergab einen Wert von 0,71 Einheiten. Die Aktivitätsbestimmung der Standardenzymlösung nach Inkubation mit einem aliquoten Teil des die AP enthaltenden Eluates ergab den theoretischen Wert von 15 Einheiten, d. h., $\mathrm{da} ß$ es durch Gelfiltration gelungen war, die Inhibitoren zu entfernen und daß der für die Galle berechnete Wert von 0,71 Einheiten der "wahren“ Aktivität der AP entspricht.

$\mathrm{Da}$ die Elimination der Hemmstoffe durch eine Gelfiltration einen größeren Aufwand an Zeit und Material erfordert, wurde auch die Abtrennung der Phosphatase durch Fällung der Gallenproteine versucht. Als geeignete Methode dafür erschien die Behandlung der Galle mit einem Methanol-Äther-Gemisch, wie sie zur Reinigung einer AP aus Rinderknochen beschrieben worden war (8). Diese Methode hat den Vorteil, daß das verwendete Fällungsmittel durch Abdampfen im Vakuum leicht entfernt werden kann. Die auf diese Art ermittelte Aktivität betrug 0,71 Einheiten. Daraus folgt, daß auch nach Fraktionierung der Galle durch dieses Verfahren die Aktivität der AP richtig bestimmt werden kann. Diese Methode hat außerdem den Vorteil, daß sehr viele Bestimmungen gleichzeitig durchgeführt werden können.

Manche Autoren bestimmen die Aktivität der AP in der Galle nach Verdünnen mit Wasser oder isotoner $\mathrm{NaCl}$ Lösung $(9,10)$. Nach Verdünnung mit Substratlösung konnte eine Aktivität von 0,47 Einheiten gemessen werden. Dieses Ergebnis zeigt, daß Verdünnung und gleichzeitige relative Erhöhung der Substratkonzentration gegenüber der Konzentration der Inhibitoren nicht ausreicht, um die Bestimmung des richtigen Wertes $\mathrm{zu}$ ermöglichen.

Tab. 1

Aktivität der alkalischen Phosphatase nach Behandlung

\begin{tabular}{lc}
\hline \multicolumn{1}{c}{ Methode } & Gefundene Einheiten \\
\hline Unbehandelte Rindergalle & 0,10 \\
Dialyse & 0,21 \\
Verdünnung $(1: 25)$ und Erhöhung & \\
der Substratkonzentration & 0,47 \\
Fällung mit Methanol-Äther & 0,71 \\
Gelfiltration & 0,71 \\
\hline
\end{tabular}

Die Tabelle 1 zeigt gemessene Aktivitäten der AP nach Anwendung der in dieser Arbeit zur Ausschaltung der Hemmstoffe verwendeten Methoden.

Der Fa. C. F. Boehringer \& Söhne GmbH, Wien, danke ich für die freundliche Überlassung mehrerer Biochemica Test-Combinationen.

\section{Literatur}

1. Strumia, E., Arch. Sci. Biol. (Bologna) 44, 337 (1960); C. A. 56, 700 (1962). - 2. Rossini, L., Boll. Soc. ital. Biol. sperim. 36, 255 (1960); C. A. 56, 3806 (1962). - 3. BrRó, J., E. GRÁsZ und M. RÉNYI-VAMOS, Z. Vitamin-, Hormon- u. Fermentforsch. 11, 290 (1961). - 4. BESSEY, O. A., H. O. LoWRY und M. J. BRock, J. biol Chemistry 164, 321 (1946). - 5. Hinsberg, K. und K. LANG, Medizinische Chemie, S. 1091, Urban und Schwarzenberg,
München (1957). - 6. Gomori, G., in: Collowick-Kaplan, Methods in Enzymology, Vol. I, S. 138, Academic Press, New York (1955). - 7. Peterlik, M., Mh. Chem. 96, 1261 (1965). 8. Volkin, E., in: Collowick-Kaplan, Methods in Enzymology, Vol. II, S. 540, Academic Press, New York 1955. - 9. NorBERG, B., Acta physiol. scand. 23, 205 (1951). - 10. LORENTZ, K., Klin. Wschr. 41, 18 (1963).
Dr. phil. M. Peterlik

Institut für allgemeine u. experimentelle Pathologie der Universität Wien Wien IX., Währinger Str. 13 (Österreich) 